\title{
Optimal Overcurrent Relays Coordination using an Improved Grey Wolf Optimizer
}

\author{
Noor Zaihah Jamal ${ }^{1}$, Mohd Herwan Sulaiman ${ }^{2}$, Omar Aliman ${ }^{3}$, Zuriani Mustaffa ${ }^{4}$ \\ Faculty of Engineering Technology ${ }^{1}$, \\ Faculty of Electrical \& Electronics Engineering ${ }^{2,3}$, \\ Faculty of Computer Systems and Software Engineering ${ }^{4}$ \\ Universiti Malaysia Pahang \\ 26300 Gambang, Pahang
}

\begin{abstract}
Recently, nature inspired algorithms (NIA) have been implemented to various fields of optimization problems. In this paper, the implementation of NIA is reported to solve the overcurrent relay coordination problem. The purpose is to find the optimal value of the Time Multiplier Setting (TMS) and Plug Setting (PS) in order to minimize the primary relays' operating time at the near end fault. The optimization is performed using the Improved Grey Wolf Optimization (IGWO) algorithm. Some modifications to the original GWO have been made to improve the candidate's exploration ability. Comprehensive simulation studies have been performed to demonstrate the reliability and efficiency of the proposed modification technique compared to the conventional GWO and some well-known algorithms. The generated results have confirmed the proposed IGWO is able to optimize the objective function of the overcurrent relay coordination problem.
\end{abstract}

Keywords-Time multiplier setting (TMS); plug setting (PS; grey wolf optimization algorithm (GWO); overcurrent relay coordination

\section{INTRODUCTION}

The electricity demand is keep increasing from year to year to accommodate the grown of the human population. In order to provide the best services, the old power system must be improved and transformed to be more compatible. Complex electrical power networking systems comprise with switchgears, transformers, ring main units and motors. All the equipment is located at different voltage rating which needs to be protected in to ensure that any fault occurrences are under control and does not affect the healthy portion of the system. To ensure the flexibility of the system to withstand any abnormal condition, the numbers of protective devices must be well arranged and coordinated.

The overcurrent relay coordination problem has been recognised as a constrained optimization problem [1-4]. Optimization of the overcurrent relay operating time (Top) is certified by two parameters which are Time Multiplier Setting (TMS) and Plug Setting (PS). These two parameters are formulated as Mix Integer Non-Linear Programming (MINLP) problem. No matter how details the progress undergoes during design stage, it is impossible to build a system without failure with external cause [5]. However, the huge catastrophe could be reduced with good and well-coordinated protection scheme. The good protection scheme should comprehend the requirements of sensitivity, speed, reliability and last but not least selectivity. Moreover, in this modern complicated electrical networking system, more numbers of relays should be coordinated.

During decade back, the implementation of analytical and graphical approach as in $[6,7]$ has been used to coordinate the overcurrent relay. The improvements of the technique have been done in [8], derivation of new non-standard tripping characteristic. In [9], a new method for repairing and inspecting curve crossing between primary and back-up relay has been developed. Meanwhile, to tackle sympathy trips threats to the system additional constraint has been introduced in [10].

Modern techniques by nature inspired have been introduced which started with Genetic Algorithm (GA) [11-14]. GA has becoming a most popular algorithm in this area in early 90s. Improvement to this algorithm have been made in [15] called Continuous Genetic Algorithm (CGA) where CGA has been proven to be faster in result generated compared to binary GA, since the chromosome in CGA does not need to be decoded. Ref. [16] has developed an improvement method to solve the mis-coordination problem which updated the weighting factors during simulation called fuzzy based Genetic Algorithm method. Next evolution of bio-nature inspired technique is introduced in [17-19] called as Particle Swarm Optimization (PSO). The PSO has been proven to provide better result compared to conventional GA and modern GA. The revolution of the algorithm is continued by Differential Evolution (DE) and Modified Differential Evolution (MDE) method as in [2022], and Invasive weed optimization [23]. In order to generate better performance of MDE, hybrid method has been developed in [3, 24-26]. Cuckoo Search Algorithm is developed in [27]. Electromagnetic Field Optimization (EFO) method in [28] and Improved GSO has been introduced in [1]. All of these algorithms are developed to search for the best overcurrent relays setting. Hybridization of some methods such as PSO-TVAC [29], GA-NLP [30], Fuzzy based-GA [31] and Hybrid PSO [32]. are also developed to improve the generated optimum results.

Recently, a new reliable and robust algorithm have been introduced known as Grey Wolf Optimization (GWO) technique. This GWO algorithm have been implemented in [33] in biomedical engineering field, optimal reactive power 
dispatch problem [34] and combined economic emission dispatch problems [35]. GWO has been introduced by [36] which is inspired by hunting behavior of a group of wolves. Some amendments have been applied to the conventional GWO in order to improve the exploration rate of the searching agents. The conventional GWO has been identified having low convergence speed and in most cases being trapped in local optimal. The recommended improvement has increased the number of searching agents instead of role as followers to the first three best agents. The objective of this paper is to pick the best TMS and PS value in order to minimize the objective function.

This paper is organized as follows; section II presents overcurrent relay coordination problem formulation. Explanation of the conventional GWO is presented in section III. Section IV explain on the improvement of the GWO algorithm. Results and analysis is presented in Section V. Finally, section VI concludes the achievements of the proposed algorithm.

\section{PROBLEM Formulation}

The coordination problem of overcurrent relays is formulated as an optimization problem. To optimize the nonlinear objective function, various nonlinear inequality constraints shall be satisfied.

\section{A. Objective Function}

The objective of the coordination problem is minimization of the primary relays' total operating time and remain the primary - backup pair relays coordinated with fulfilled the $0.2 \mathrm{~s}$ $-0.5 \mathrm{~s}$ coordination time interval (CTI). The minimization of the relay's operating time is close related to the optimization of the value of TMS and PS. The objective function is.

$$
\min F=\sum_{i=1}^{n} \omega_{i} T_{i}
$$

Where $\omega_{i}$ is the weight of relay $R_{i}$ and $n$ is the number of relays inside the system. While $T_{i}$ is the operating time of primary relay. Generally the value of $\omega_{i}$ is set as one [30, 37], hence (1) becomes:

$\min F=\sum_{i=1}^{n} T_{i}$

The relay operating time is define by IEC standard [38] as

$$
T_{i}=T M S_{i} \times \frac{k}{\left(\frac{I_{s c}}{P S_{i}}\right)^{\alpha}-1}
$$

Where $P S_{i}$ is plug setting for relay $R_{i}, T M S_{i}$ is time multiplier setting for relay $R_{i}, I_{s c}$ is short circuit current which seen by relay $R_{i}$

\section{B. Constraints}

The objective function is possible to be achieved if relay parameters contraints and coordination constraints are fullfilled.

The relay parameters constraints are $T M S$ and $P S$ boundaries

$$
P S_{i}^{\min } \leq P S_{i} \leq P S_{i}^{\max }
$$

The boundary of the PS can be calculated as

$$
\begin{aligned}
& P S_{\text {min }}=1.25 \times I_{n} \\
& P S_{\text {max }}=\frac{2}{3} \times I_{f \text { min }}
\end{aligned}
$$

Where $I_{n}$ is the normal current rating which protected by the relay $R_{i} . I_{f \min }$ is the minimum value of current which is detected as fault by relay $R_{i}$

The boundary of TMS is given as

$$
T M S_{i}^{\min } \leq T M S_{i} \leq T M S_{i}^{\max }
$$

The TMS value is the time delay that varies from 0.1 to 1.1 $[3,4]$. Where $T M S_{\text {min }}$ is minimum limit and $T M S_{\text {max }}$ is maximum limit value of TMS for relay $R_{i}$

The coordination constraints is in between Back-up and Primary relay. The selectivity should fullfilled the time interval required. The primary relay should reacted in advanced during fault occurences as compared to back-up relay and not vise versa to escape any sympathy trips

$$
C T I=T_{b c}-T_{p r}
$$

Where $T_{p r}$ is primary relay time operating, $T_{b c}$ is the backup relay time operating and CTI varies between $0.2 \mathrm{~s}-0.5 \mathrm{~s}$ [3].

\section{GREY WOLF OPTIMIZATION ALGORITHM}

This section presents an overview of the conventional grey wolf optimization algorithm. Details on the GWO can be found in [36]. Then, in the next section the improvement to the proposed algorithm will be presented.

\section{A. Conventional Grey Wolf Optimization Algorithm}

The Grey Wolf Optimizer is derived by leadership hierarchy and hunting of grey wolf. The dominant social hierarchy of grey wolf have an average group of 5-12 members. The first tier called alpha $(\alpha)$ which dominating the group and responsible for decisions making as a leader. The dominant alpha is selected based on ability to manage their group members well.

The next tier is called beta $(\beta)$ role as assistance to alpha in order to enforce any instruction or command by the leader. Beta could be the next leader with good discipline criteria which can be either male or female. 


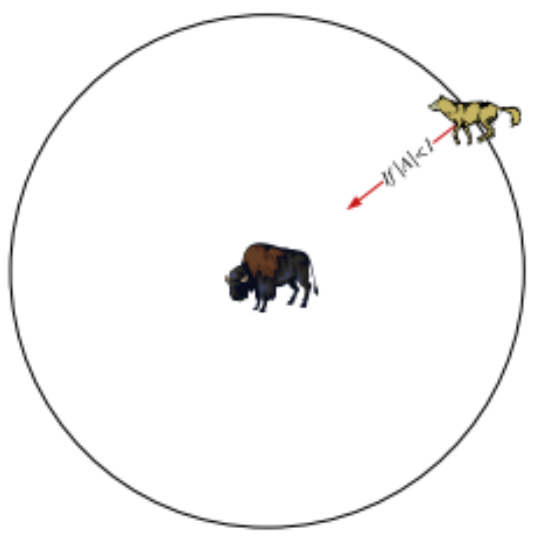

Fig. 1. Attacking the Prey [36].

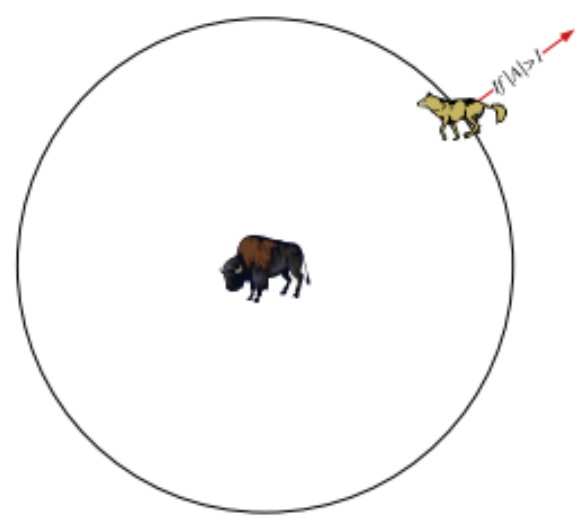

Fig. 2. Diverge from the Prey [36].

Delta $(\delta)$ is once used to be beta and alpha would be placed on the third-tier roles as hunters, caretakers to the younger members, sentinels and scouts. Hunters help foods delivering to the group members. Caretakers take care of the weak, ill and wounded young members. Sentinels control the security of the members and guarantee their territory safety and scouts role as territory marker to monitor the boundaries and discover any dangers ahead.

The bottom ranking is Omega $(\omega)$. Omega appears to be a balance to the nature bio-chain of the grey wolf. Even though their existence is not really appreciated by the other members of the group but still their role as a babysitter to the group can be acceptable. They are last wolves that are permitted to eat the prey.

In grey wolf community, the hunting activity is categorized by three phases as follows:

- Tracking: trace the location of the prey.

- Encircling: trap the prey in a circle.

- Attacking: move towards the prey by fulfilling the terms.

Alpha will lead during the hunting activities as the best solution, followed by Beta as second best and Delta as the third best. Omega will update positions as remaining solution by considering the position of the first, second and third best of the group.

For mathematical encircling activity behaviour modelling, below equation is considered [36]:

$$
\begin{aligned}
& \vec{D}=\left|\vec{C} \cdot \vec{X}_{p}(t)-\vec{X}(t)\right| \\
& \vec{X}(t+1)=\vec{X}_{p}(t)-\vec{A} \cdot \vec{D}
\end{aligned}
$$

Where $\vec{X}_{p}$ is the position of the prey, $\vec{X}$ is the grey wolf position vector, $\vec{C}$ and $\vec{A}$ are vector's coefficient and $t$ is the present iteration. The formulation of the vector's coefficient are as following equation [36]:

$$
\begin{aligned}
& \vec{A}=2 \vec{a} \cdot \vec{r}_{1}-\vec{a} \\
& \vec{C}=2 \cdot \vec{r}_{2}
\end{aligned}
$$

According to grey wolf hunting behavior, they will repositioning their current location according to the position of the prey. The value of vector $\vec{A}$ and $\vec{C}$ will be the updated position with respect to the current position of the wolf which means, adjusting the value of $\vec{A}$ and $\vec{C}$ can placed the wolf to the different places. where $\vec{a}$ are linearly reduced from 2 to 0 over the iterations course, $\vec{r}_{1}$ and $\vec{r}_{2}$ are random vectors within $(0,1)$. The random value of $\vec{r}_{1}$ and $\vec{r}_{2}$ allows agents to move to any position around the prey in random location by using eq. (12) and (13).

It is tough to locate the prey's location furthermore in an open search area. For mathematical hunting activity modelling purposes, the alpha, beta and delta are assumed to have knowledge on the prey's location based on their bio-nature capabilities. Therefore, the first solution of $\alpha, \beta$ and $\delta$ force the remaining search agents (including $\omega$ ) to update their locations by referring according to the location of the best search agents [36].

The following formulas are obtained.

$$
\begin{aligned}
& \vec{D}_{i}=\left|\vec{C}_{n} \cdot \vec{X}_{i}-\vec{X}\right| \\
& \vec{X}_{n}=\vec{X}_{i}-\vec{A}_{n} \cdot\left(\vec{X}_{i}\right)
\end{aligned}
$$
3.

Where $i$ indicate the search agent of $\alpha, \beta$ and $\delta$ and $n=1,2$,

$$
\vec{X}(t+1)=\frac{\vec{X}_{m}+\vec{X}_{m+1}+\vec{X}_{m+2}}{3}
$$

Where $t$ is the present iteration and $m=1$ which indicate the updated position of the $\alpha, \beta$ and $\delta$. 
The random position within the search area is updated according to the first three best solutions. The estimated position of the prey by alpha, beta and delta will then be a guide to omegas to update their positions.

The last stage of hunting is by attacking when the prey is in static position. The decreasing value of $\vec{a}$ is when the wolves are approaching the prey. This will also decrease the value of $\vec{A}$ which $\vec{A}$ is a random value between (-2a, $2 \mathrm{a})$. The wolves are moving forward to attack the prey if $|A|<1$ as in fig. 1 and fig. 2. The process is repeating for the next iteration until the termination criterion is justified.

\section{B. Improved Grey Wolf Optimization (IGWO)}

The most challenging task in bio-nature population is to avoid the searching agents from trapping inside the local optimal. The end result of the objective function is influenced by this trapping problem and only near optimal solution is generated. The converging towards global optimal could be segregated in two different conditions. At the first place, the searching agents should be motivated to disperse throughout the wide range of searching space to find out the potential prey instead of crowding around the consistent local optimal. This stage also called as exploration stage. In the next stage which called exploitation stage, where the searching agents should be able to manipulate the knowledge of the potential prey to converge towards the global optimal value. In GWO, fine tuning of the parameters $\vec{a}$ and $\vec{A}$ could balance these two stages.

From the eq. (12), the coefficient vector of $\vec{A}$ is influence by component $\vec{a}$ with the formulation as follows [36]:

$\vec{a}=2-1 *\left(\frac{2}{\max \text { iter }}\right)$

Some recommendation by researchers' that the exploration stage motivates the searching agents to update their position stochastically and abruptly. This situation has improved the variety of the solution and resulted to increase exploration wisdom in the search space.

But on the other hand, the exploitation is focusing on improving the solution's quality by searching locally around the promising area. In this stage, the search agents are obliged to search locally.

In general, the probability of the local optimal trapped could be avoided with the wisdom of explorations by the searching candidates. In conventional GWO, tracking or hunting activity is only considered the knowledge of the alpha, beta and delta whereas the rest wolves are obliged to follow them including omega.

In order to increase the exploration wisdom of the search agents, some modification to the conventional GWO algorithm has been recommended. Improved GWO (IGWO) algorithm proposed that omega should be considered as a searching agent instead of obliged to follow the first three best candidates. The increasing of the numbers of searching agents improve the search ability of the grey wolves in a wide range of search space. This improvement motivates the search agents to be scattered during exploration stage. In other words, that the wide range of the search space could be explored in further by the increasing of the search agents. The hunting activity could be more efficient and time saving. The mathematical modelling of the IGWO hunting agents are as follows:

$$
\begin{aligned}
& \vec{D}_{\alpha}=\left|\vec{C}_{1} \cdot \vec{X}_{\alpha}-\vec{X}\right| \\
& \vec{D}_{\beta}=\left|\vec{C}_{2} \cdot \vec{X}_{\beta}-\vec{X}\right| \\
& \vec{D}_{\delta}=\left|\vec{C}_{3} \cdot \vec{X}_{\delta}-\vec{X}\right| \\
& \vec{D}_{\omega}=\left|\vec{C}_{4} \cdot \vec{X}_{\omega}-\vec{X}\right| \\
& \vec{X}_{1}=\vec{X}_{\alpha}-\vec{A}_{1} \cdot\left(\vec{X}_{\alpha}\right) \\
& \vec{X}_{2}=\vec{X}_{\beta}-\vec{A}_{2} \cdot\left(\vec{X}_{\beta}\right) \\
& \vec{X}_{3}=\vec{X}_{\delta}-\vec{A}_{3} \cdot\left(\vec{X}_{\delta}\right) \\
& \vec{X}_{4}=\vec{X}_{\omega}-\vec{A}_{4} \cdot\left(\vec{X}_{\omega}\right) \\
& \vec{X}_{(t+1)}=\frac{\vec{X}_{1}+\vec{X}_{2}+\vec{X}_{3}+\vec{X}_{4}}{4}
\end{aligned}
$$

In [39], it is argued that, too much exploration will have resulted to too much randomness and probably generates bad results. However, this argument could be counteracted by the increased numbers of the active exploration agents. The flow chart of the application of IGWO to relay coordination problem as fig. 3. The pseudocode of the IGWO as in fig. 4

Initialized the search agents' population $X i(i=1,2 \ldots, n)$
Initialized the $a, A$ and $C$
Calculate the fitness of each search agent
$X_{\alpha}=$ the best search agent
$X_{\beta}=$ the second best search agent
$X_{\delta}=$ the third best search agent
while ( $t<$ max iteration no.)
for each search agent
Update the position of current search agent by (26)
end for
Update $a, A$ and $C$
Calculate the fitness of the search agents
Update $X_{a}, X_{\beta}, X_{b}$ and $X_{\omega}$
$t=t+1$
end while
return $X_{\alpha}$

Fig. 3. Pseudo Code of IGWO Algorithm. 


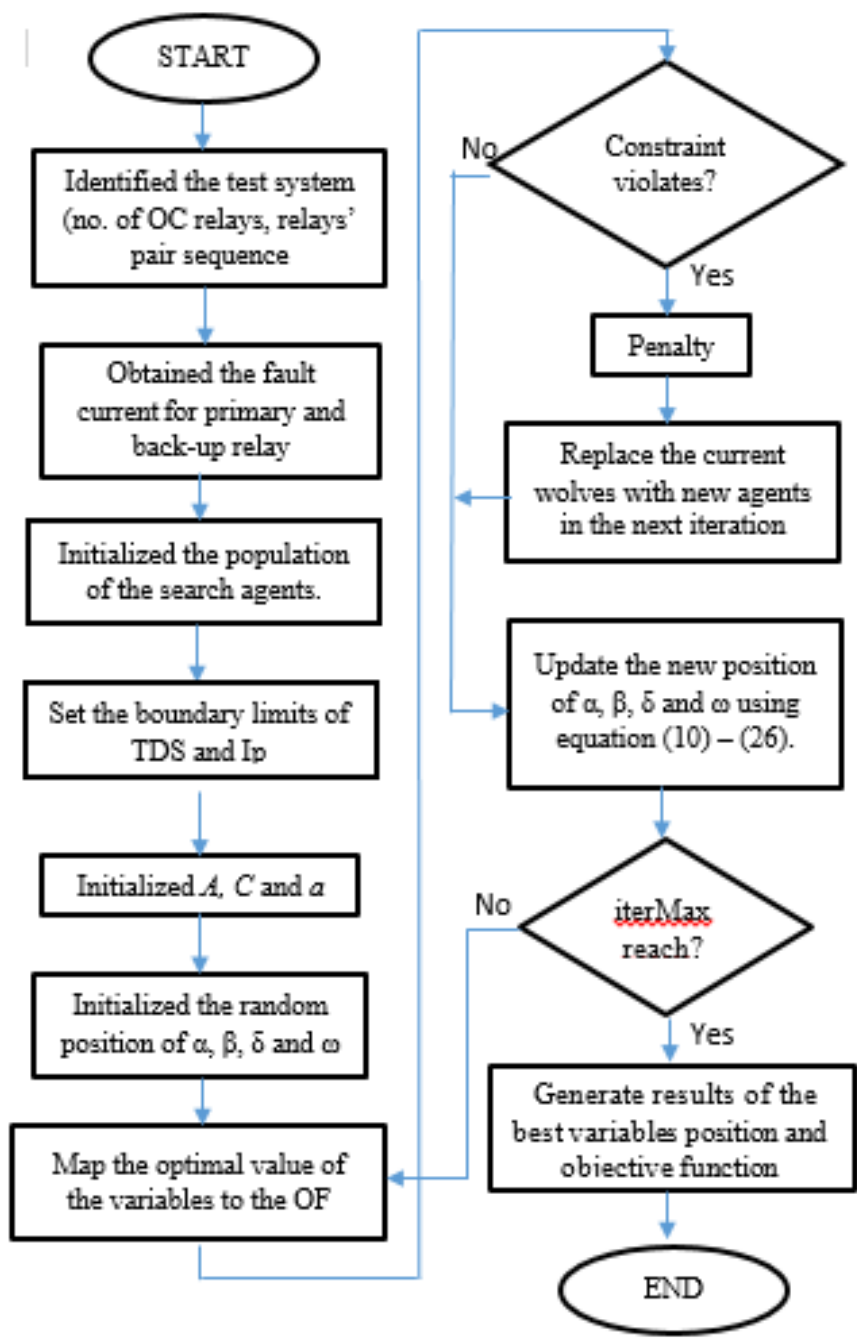

Fig. 4. Implementation of IGWO to Coordination Overcurrent Relay Problem.

\section{RESULTS AND DISCUSSIONS}

Simulations have been performed to three different IEEE test cases (three-bus, eight-bus and 15-bus test system) to test the efficiency of the GWO and IGWO techniques. The simulations are using MATLAB software and executed on an intel core i5-6200U CPU, $2.3 \mathrm{GHz}$ with 8GB RAM. The implemented value of CTI is 0.2 to $0.5 \mathrm{~s}$. The constant values used are according to IEC standard [38] and implemented normal inverse characteristic to all of the test case where with $\mathrm{k}$ $=0.14$ and $\alpha=0.02$

\section{A. Case I}

The system consists of three busbar $\left(\mathrm{B}_{1}, \mathrm{~B}_{2}\right.$ and $\left.\mathrm{B}_{3}\right)$, six overcurrent relay $\left(R_{1}, R_{2}, \ldots R_{6}\right)$, three ring lines and powered by three generators with $69 \mathrm{kV}$ system voltage. The TMS and PS are considered as variables which bound from $X_{1}$ to $X_{6}$ and $\mathrm{X}_{7}$ to $\mathrm{X}_{12}$ respectively.

The results are presented in MINLP with continuous TMS and PS models for this case study. The search agents are 30 and iteration no. implemented is 1000 .
TABLE I. COMPARISON OF IGWO WITH OTHERS TECHNIQUE FOR CASE I

\begin{tabular}{|l|l|}
\hline Method & Objective function (s) \\
\hline Modified PSO [2] & 1.9258 \\
\hline MINLP [4] & 1.727 \\
\hline Seeker Algorithm [4] & 1.599 \\
\hline GWO & 1.5124 \\
\hline IGWO & 1.4789 \\
\hline
\end{tabular}

TABLE II. COMPARISON OF IGWO WITH OTHERS TECHNIQUE FOR CASE I

\begin{tabular}{|l|l|l|l|l|l|}
\hline \multirow{2}{*}{ Relay no. } & \multirow{2}{*}{ CT } & \multicolumn{3}{|l|}{ GWO } & \multicolumn{2}{l|}{ IGWO } \\
\cline { 3 - 6 } & & TMS & PS & TMS & PS \\
\hline 1 & 300 & 0.1000 & 3.0 & 0.1000 & 1.5000 \\
\hline 2 & 200 & 0.1001 & 1.5 & 0.1000 & 2.6166 \\
\hline 3 & 200 & 0.1000 & 3.0 & 0.1001 & 2.9770 \\
\hline 4 & 300 & 0.1000 & 3.0 & 0.1000 & 1.5858 \\
\hline 5 & 200 & 0.1001 & 1.5 & 0.1000 & 2.8169 \\
\hline 6 & 400 & 0.1000 & 1.5 & 0.1000 & 1.5009 \\
\hline Result (s) & & 1.5124 & & 1.4789 & \\
\hline
\end{tabular}

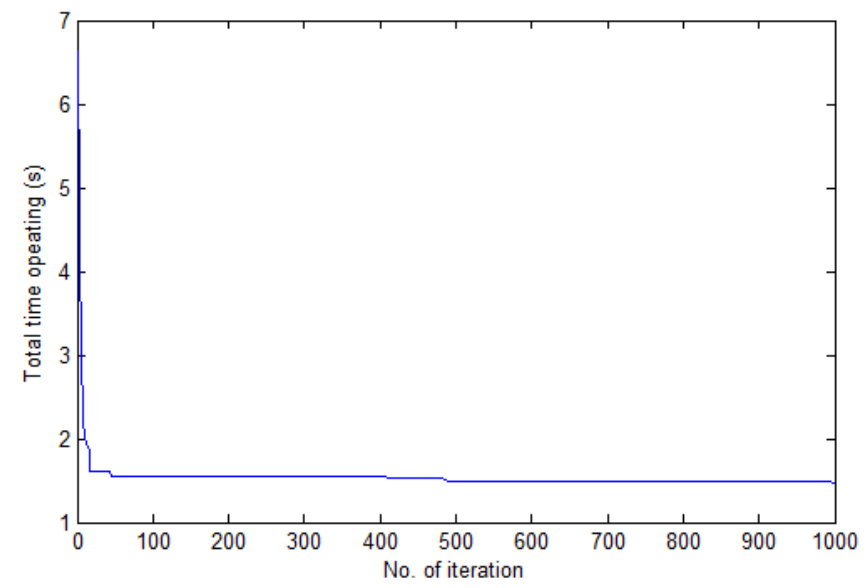

Fig. 5. Best Result for 1000 Iteration.

In [40], the details of this test case can be obtained. The TMS values is bound from 0.1 s to $1.1 \mathrm{~s}[3,4]$ and the PS values bound from 1.5 to 5[4]. The CTI value of $0.3 \mathrm{~s}$ is applied to this three bus test case.

Table I shows the comparative results of the IGWO with the modified PSO, MINLP, Seeker Algorithm and conventional GWO. The optimized result of conventional GWO and IGWO are shown in Table II. From table II, it can be seen that the IGWO performs better solution with $0.0335 \mathrm{~s}$ faster than GWO. This has proven that improvement of GWO performs the best way compared to the others technique applied before. Fig. 5 shows the generated best solution for 1000 iteration with 30 agents.

The best result in fig.5 has shown the efficiency of the IGWO in 30 free running conditions while in fig. 6 , the convergence of the mean and best result is presented. 


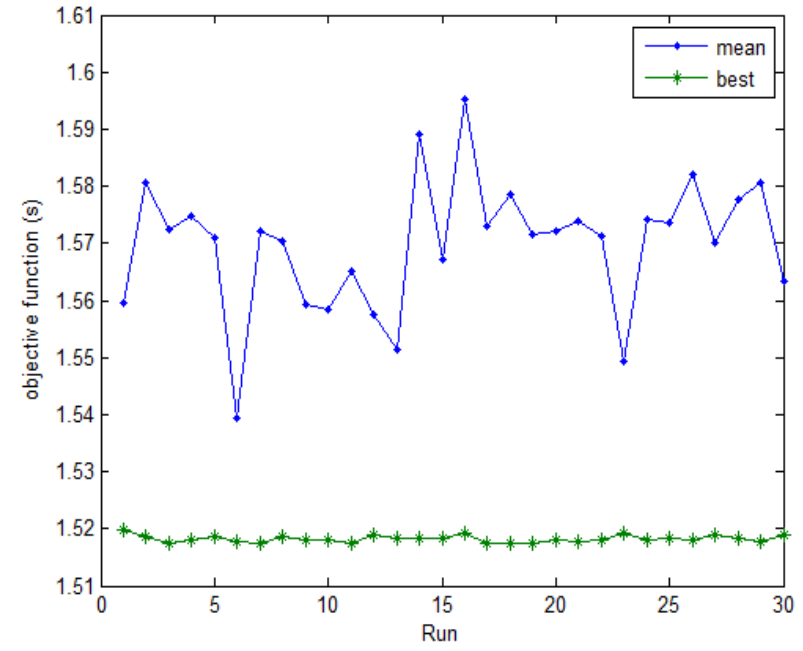

Fig. 6. Performance of IGWO for 30 Free Running.

\section{B. Case II}

The case 2 consist of 14 overcurrent relays $\left(R_{1}, R_{2}, \ldots R_{14}\right)$, seven ring lines to connect six busbars $\left(\mathrm{B}_{1}, \mathrm{~B}_{2} \ldots \ldots \mathrm{B}_{6}\right)$ as in fig. 7. The bound of TMS value from $X_{1}$ to $X_{14}$ and bound of PS is from $\mathrm{X}_{15}$ to $\mathrm{X}_{28}$. The dimension of variables is 28 with constraints of 20 .

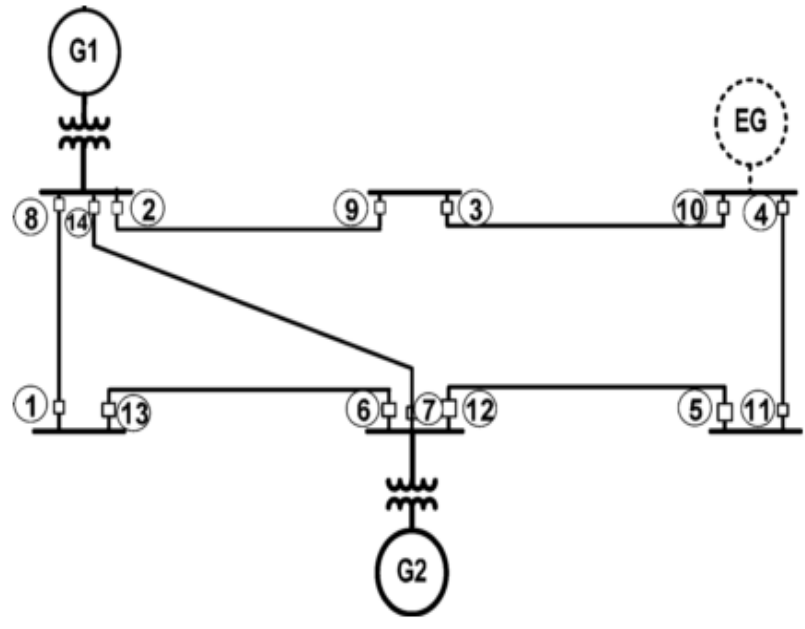

Fig. 7. Test Case 2 with IEEE 8 Bus System.

The TMS values are varies in between $0.1 \mathrm{~s}$ to $1.1 \mathrm{~s}$ and the PS values are in between 1.5 to 5. Both TMS and PS are continuous models. The current transformer ratio of each relays are as stated in table III. The details of this test system can be obtained from [19].

The comparative results of the IGWO with GA-NLP, CSA, Seeker Algorithm and conventional GWO are tabulated in Table III.

Table IV shows the optimized value of TMS and PS for conventional GWO and IGWO. Based on the obtained results, the IGWO has decreased the total operating time of the primary relays around $0.0114 \mathrm{~s}$ compared to conventional GWO.
TABLE III. COMPARISON OF IGWO WITH OTHERS TECHNIQUE FOR CASE II

\begin{tabular}{|l|l|}
\hline Method & Objective function (s) \\
\hline GA-NLP [30] & 10.9499 \\
\hline CSA [41] & 9.7628 \\
\hline Seeker Algorithm [4] & 8.4270 \\
\hline GWO & 8.0979 \\
\hline IGWO & 8.0865 \\
\hline
\end{tabular}

TABLE IV. OPTIMUM SETTING FOR CASE II

\begin{tabular}{|c|c|c|c|c|c|}
\hline \multirow{2}{*}{$\begin{array}{l}\text { Relay } \\
\text { no. }\end{array}$} & \multirow{2}{*}{ CT } & \multicolumn{2}{|l|}{ GWO } & \multicolumn{2}{|l|}{ IGWO } \\
\hline & & TMS & PS & TMS & PS \\
\hline 1 & 1200 & 0.1001 & 1.5014 & 0.1000 & 1.5000 \\
\hline 2 & 1200 & 0.1101 & 4.1984 & 0.1000 & 4.4101 \\
\hline 3 & 800 & 0.1759 & 4.9241 & 0.1722 & 4.9957 \\
\hline 4 & 1200 & 0.4346 & 5.0000 & 0.435 & 5.0000 \\
\hline 5 & 1200 & 0.1015 & 1.5158 & 0.1001 & 1.5082 \\
\hline 6 & 1200 & 0.1008 & 1.5214 & 0.1015 & 1.5048 \\
\hline 7 & 800 & 0.1003 & 1.5801 & 0.1055 & 1.9719 \\
\hline 8 & 1200 & 0.1010 & 4.3769 & 0.1004 & 4.4778 \\
\hline 9 & 800 & 0.1016 & 4.6648 & 0.1000 & 4.7092 \\
\hline 10 & 1200 & 0.1008 & 4.1119 & 0.1001 & 4.2285 \\
\hline 11 & 1200 & 0.1005 & 1.8435 & 0.1015 & 1.6762 \\
\hline 12 & 1200 & 0.1000 & 2.1878 & 0.1011 & 2.2784 \\
\hline 13 & 1200 & 0.1001 & 1.6084 & 0.1052 & 1.5139 \\
\hline 14 & 800 & 0.1046 & 2.0965 & 0.1015 & 2.0523 \\
\hline $\begin{array}{l}\text { Result } \\
\text { (s) }\end{array}$ & & 8.0979 & & 8.0865 & \\
\hline
\end{tabular}

Table IV shows that IGWO has outperform others optimization algorithm for this test case. This proves that IGWO has better efficiency towards conventional GWO and other identified algorithm.

\section{Case III}

In this case, the proposed method is applied to IEEE 15 bus test system. The system's single line diagram is as in Fig. 8. The system details on three phase short circuit data can be found in [2]. This system is powered by highly distributed generation network with 15 bus consists of 42 relays and connected by 21 lines. 


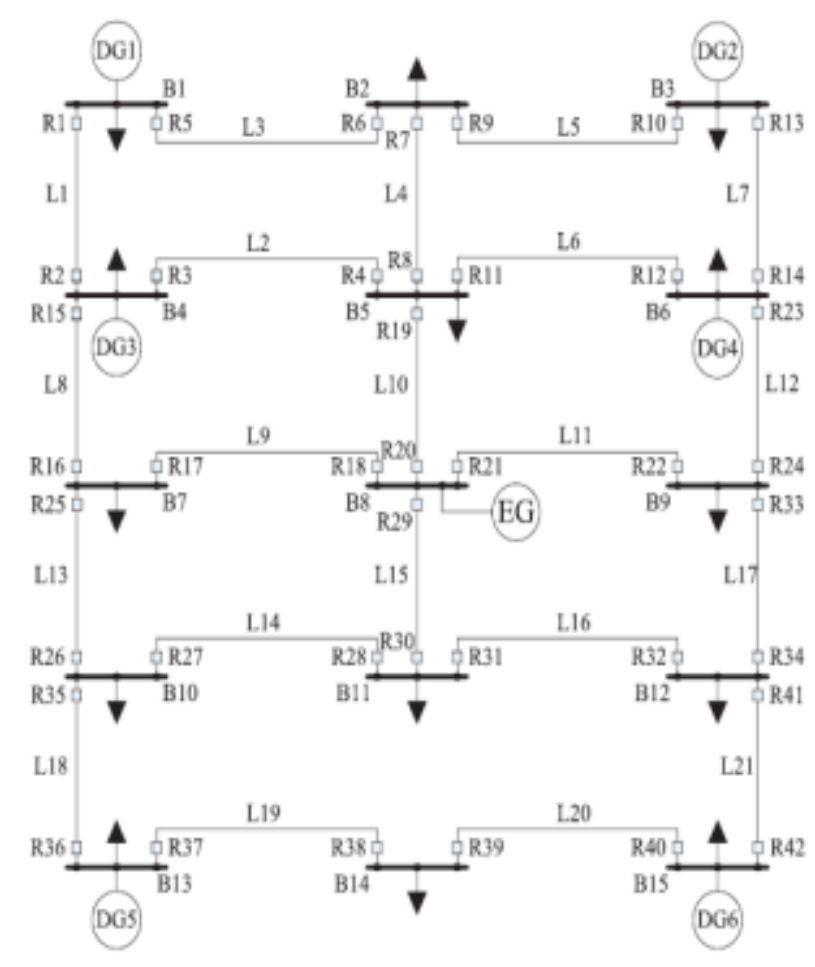

Fig. 8. IEEE 15 Bus Test System.

TABLE V. COMPARISON OF IGWO WITH OTHERS TECHNIQUE FOR CASE III

\begin{tabular}{|l|l|}
\hline Method & Objective function (s) \\
\hline GA-NLP [30] & 19.5843 \\
\hline CSA [41] & 19.5521 \\
\hline PSO-LP [25] & 15.0020 \\
\hline GWO & 12.9637 \\
\hline IGWO & 12.6446 \\
\hline
\end{tabular}

There are 84 variables with 82 coordination constraints. The TMS bound from $X_{1}$ to $X_{42}$ and PS bound from $X_{43}$ to $X_{84}$. The normal inverse type characteristic is selected. The TMS values are in between $0.1 \mathrm{~s}$ to $1.1 \mathrm{~s}$ and the PS value is in between 1.5 to 5 . The CTI value is assumed as $0.2 \mathrm{~s}$.

Table $\mathrm{V}$ shows the comparative results of the IGWO with GA-NLP, CSA, PSO-LP and conventional GWO. From the generated results, it has confirmed the robustness of IGWO in order to solve the optimization problem of overcurrent relay coordination.

TABLE VI. PERFORMANCE COMPARISON FOR GWO AND IGWO FOR CASE III

\begin{tabular}{|l|l|l|}
\hline Criteria & GWO & IGWO \\
\hline Best & 12.9637 & 12.6446 \\
\hline Worst & 82.0985 & 81.6864 \\
\hline Mean & 17.7500 & 17.6599 \\
\hline
\end{tabular}

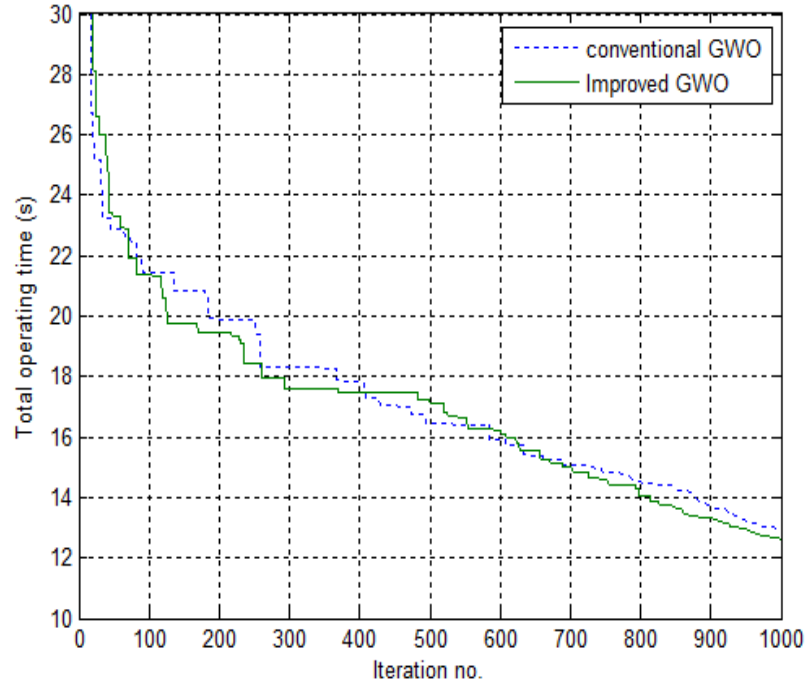

Fig. 9. Conventional Versus Improved GWO for Case III

TABLE VII. OPTIMUM SETTING FOR 15 BUS TEST CASE

\begin{tabular}{|c|c|c|c|c|}
\hline \multirow{2}{*}{ Relay no. } & \multicolumn{2}{|l|}{ GWO } & \multicolumn{2}{|l|}{ IGWO } \\
\hline & TMS & PS & TMS & PS \\
\hline 1 & 0.1013 & 2.7320 & 0.1001 & 2.8114 \\
\hline 2 & 0.1001 & 1.7947 & 0.1000 & 1.5104 \\
\hline 3 & 0.1002 & 1.5621 & 0.1048 & 1.7950 \\
\hline 4 & 0.1018 & 2.6221 & 0.1002 & 3.0701 \\
\hline 5 & 0.1083 & 1.5487 & 0.1003 & 1.5191 \\
\hline 6 & 0.1002 & 1.5042 & 0.1004 & 1.5367 \\
\hline 7 & 0.1013 & 2.1928 & 0.1000 & 2.4252 \\
\hline 8 & 0.1001 & 2.5192 & 0.1012 & 2.3699 \\
\hline 9 & 0.1004 & 2.0425 & 0.1030 & 2.0256 \\
\hline 10 & 0.1003 & 1.7860 & 0.1000 & 1.5244 \\
\hline 11 & 0.1000 & 2.5195 & 0.1002 & 2.5299 \\
\hline 12 & 0.1012 & 2.8016 & 0.1008 & 2.8068 \\
\hline 13 & 0.1003 & 2.7715 & 0.1004 & 2.4412 \\
\hline 14 & 0.1033 & 1.6329 & 0.1001 & 1.6608 \\
\hline 15 & 0.1011 & 1.6711 & 0.1000 & 1.5666 \\
\hline 16 & 0.1007 & 1.5035 & 0.1001 & 1.7755 \\
\hline 17 & 0.1003 & 1.9094 & 0.1008 & 1.5505 \\
\hline 18 & 0.1003 & 2.0602 & 0.1130 & 1.6783 \\
\hline 19 & 0.1001 & 2.1253 & 0.1003 & 2.1283 \\
\hline 20 & 0.1002 & 1.6312 & 0.1040 & 1.5726 \\
\hline 21 & 0.1001 & 2.4752 & 0.1002 & 2.0357 \\
\hline
\end{tabular}

Table VI and VIII provide the optimum setting of TMS and PS respectively. From the tabulated results, it indicates that IGWO has outperformed about $0.3191 \mathrm{~s}$ faster than the results of GWO. The characteristic of conventional GWO versus IGWO as in fig. 9 which shows that the IGWO has given improved result. Table VII shows the performance comparative results in between GWO and IGWO. 
TABLE VIII. OPTIMUM SETTING FOR 15 BUS TEST CASE, CONTINUED

\begin{tabular}{|c|c|c|c|c|}
\hline \multirow{2}{*}{ Relay no. } & \multicolumn{2}{|l|}{ GWO } & \multicolumn{2}{|l|}{ IGWO } \\
\hline & TMS & PS & TMS & PS \\
\hline 22 & 0.1007 & 1.5455 & 0.1001 & 1.5461 \\
\hline 23 & 0.1016 & 1.5723 & 0.1000 & 1.5502 \\
\hline 24 & 0.1000 & 1.5245 & 0.1002 & 1.6656 \\
\hline 25 & 0.1001 & 1.5056 & 0.1002 & 1.5019 \\
\hline 26 & 0.1002 & 3.1433 & 0.1002 & 2.9163 \\
\hline 27 & 0.1057 & 1.9240 & 0.1002 & 2.1045 \\
\hline 28 & 0.1007 & 1.5607 & 0.1002 & 1.5029 \\
\hline 29 & 0.1071 & 1.6619 & 0.1017 & 1.6389 \\
\hline 30 & 0.1001 & 2.1626 & 0.1006 & 2.1199 \\
\hline 31 & 0.1119 & 1.8264 & 0.1000 & 2.0376 \\
\hline 32 & 0.1005 & 2.9962 & 0.1008 & 3.0167 \\
\hline 33 & 0.1000 & 1.5108 & 0.1012 & 1.5459 \\
\hline 34 & 0.1006 & 1.9720 & 0.1006 & 1.5149 \\
\hline 35 & 0.1000 & 2.0547 & 0.1002 & 1.9585 \\
\hline 36 & 0.1003 & 3.3072 & 0.1002 & 3.3271 \\
\hline 37 & 0.1034 & 2.0512 & 0.1016 & 1.7971 \\
\hline 38 & 0.1009 & 2.4801 & 0.1002 & 2.5855 \\
\hline 39 & 0.1086 & 2.3918 & 0.1004 & 2.7681 \\
\hline 40 & 0.1001 & 2.6924 & 0.1010 & 2.5714 \\
\hline 41 & 0.1007 & 1.5155 & 0.1003 & 1.7723 \\
\hline 42 & 0.1001 & 3.6070 & 0.1001 & 3.4527 \\
\hline Result (s) & \multicolumn{2}{|l|}{12.9637} & \multicolumn{2}{|l|}{12.6446} \\
\hline
\end{tabular}

\section{CONCLUSION}

This paper proposed IGWO algorithm for optimal coordination setting of the overcurrent relays problem. Some modification has been recommended to improve the exploration ability of the grey wolves. This exploration ability has been proven to improve the conventional GWO convergence characteristic. Three test cases are utilized to confirm the reliability of the IGWO. Comparison results between IGWO, conventional GWO and with other identified algorithm such GA-NLP and CSA indicated that IGWO has improved the convergence performance when applied to the optimization problem of overcurrent relays coordination. In addition, proposed modification has counteracted the argument of randomness exploration activity. As the conclusion, the IGWO is appears to be an efficient and robust optimization algorithm for optimal solution of overcurrent relay coordination problem in electrical network system.

\section{ACKNOWLEDGMENT}

This work was supported by Universiti Malaysia Pahang under grant no. RDU1803101.

\section{REFERENCES}

[1] M. Alipour, S. Teimourzadeh, and H. Seyedi, "Improved group search optimization algorithm for coordination of directional overcurrent relays," Swarm and Evolutionary Computation, vol. 23, pp. 40-49, 8// 2015.

[2] M. M. Mansour, S. F. Mekhamer, and N. El-Kharbawe, "A Modified Particle Swarm Optimizer for the Coordination of Directional Overcurrent Relays," IEEE Transactions on Power Delivery, vol. 22, pp. 1400-1410, 2007.
[3] A. S. Noghabi, J. Sadeh, and H. R. Mashhadi, "Considering Different Network Topologies in Optimal Overcurrent Relay Coordination Using a Hybrid GA," IEEE Transactions on Power Delivery, vol. 24, pp. 18571863, 2009.

[4] T. Amraee, "Coordination of Directional Overcurrent Relays Using Seeker Algorithm," IEEE Transactions on Power Delivery, vol. 27, pp. 1415-1422, 2012.

[5] M. H. Hussain, S. R. A. Rahim, and I. Musirin, "Optimal Overcurrent Relay Coordination: A Review," Procedia Engineering, vol. 53, pp. 332336, // 2013.

[6] C. W. Rogers, R. Ramaswami, and P. F. McGuire, "Graphical coordination program aids system relaying at Consumers Power," IEEE Computer Applications in Power, vol. 3, pp. 30-35, 1990.

[7] "Computer Representation of Overcurrent Relay Characteristics," IEEE Power Engineering Review, vol. 9, pp. 50-51, 1989.

[8] T. Keil and J. Jager, "Advanced Coordination Method for Overcurrent Protection Relays Using Nonstandard Tripping Characteristics," Power Delivery, IEEE Transactions on, vol. 23, pp. 52-57, 2008.

[9] Y. Lu and J.-L. Chung, "Detecting and solving the coordination curve intersection problem of overcurrent relays in subtransmission systems with a new method," Electric Power Systems Research, vol. 95, pp. 1927, 2// 2013.

[10] D. Birla, R. P. Maheshwari, and H. O. Gupta, "An Approach to Tackle the Threat of Sympathy Trips in Directional Overcurrent Relay Coordination," Power Delivery, IEEE Transactions on, vol. 22, pp. 851$858,2007$.

[11] L. Cheng-Hung and C. Chao-Rong, "Using Genetic Algorithm for Overcurrent Relay Coordination in Industrial Power System," in Intelligent Systems Applications to Power Systems, 2007. ISAP 2007. International Conference on, 2007, pp. 1-5.

[12] C. W. So, K. K. Li, K. T. Lai, and K. Y. Fung, "Application of genetic algorithm for overcurrent relay coordination," in Developments in Power System Protection, Sixth International Conference on (Conf. Publ. No. 434), 1997, pp. 66-69.

[13] C. W. So, K. K. Li, K. T. Lai, and K. Y. Fung, "Application of genetic algorithm to overcurrent relay grading coordination," in Advances in Power System Control, Operation and Management, 1997. APSCOM-97. Fourth International Conference on (Conf. Publ. No. 450), 1997, pp. 283287 vol.1.

[14] F. Razavi, H. A. Abyaneh, M. Al-Dabbagh, R. Mohammadi, and H. Torkaman, "A new comprehensive genetic algorithm method for optimal overcurrent relays coordination," Electric Power Systems Research, vol. 78, pp. 713-720, 4// 2008.

[15] P. P. Bedekar and S. R. Bhide, "Optimum coordination of overcurrent relay timing using continuous genetic algorithm," Expert Systems with Applications, vol. 38, pp. 11286-11292, 9// 2011.

[16] D. S. Alkaran, M. R. Vatani, M. J. Sanjari, G. B. Gharehpetian, and M. S. Naderi, "Optimal Overcurrent Relay Coordination in Interconnected Networks by Using Fuzzy-Based GA Method," IEEE Transactions on Smart Grid, vol. PP, pp. 1-1, 2016.

[17] J. C. Bansal and K. Deep, "Optimization of directional overcurrent relay times by particle swarm optimization," in Swarm Intelligence Symposium, 2008. SIS 2008. IEEE, 2008, pp. 1-7.

[18] Z. Moravej, M. Jazaeri, and M. Gholamzadeh, "Optimal coordination of distance and over-current relays in series compensated systems based on MAPSO," Energy Conversion and Management, vol. 56, pp. 140-151, 2012.

[19] H. H. Zeineldin, E. F. El-Saadany, and M. M. A. Salama, "Optimal coordination of overcurrent relays using a modified particle swarm optimization," Electric Power Systems Research, vol. 76, pp. 988-995, 7// 2006.

[20] R. Thangaraj, M. Pant, and K. Deep, "Optimal coordination of overcurrent relays using modified differential evolution algorithms," Engineering Applications of Artificial Intelligence, vol. 23, pp. 820-829, $8 / / 2010$.

[21] R. Thangaraj, M. Pant, and A. Abraham, "New mutation schemes for differential evolution algorithm and their application to the optimization of directional over-current relay settings," Applied Mathematics and Computation, vol. 216, pp. 532-544, 3/15/ 2010. 
[22] M. Singh, B. K. Panigrahi, A. R. Abhyankar, and S. Das, "Optimal coordination of directional over-current relays using informative differential evolution algorithm," Journal of Computational Science, vol. 5, pp. 269-276, 3// 2014.

[23] C. A. Castillo, A. Conde, and M. Y. Shih, "Improvement of nonstandardized directional overcurrent relay coordination by invasive weed optimization," Electric Power Systems Research, vol. 157, pp. 48-58, 2018/04/01/2018

[24] J. A. Sueiro, E. Diaz-Dorado, E. Míguez, and J. Cidrás, "Coordination of directional overcurrent relay using evolutionary algorithm and linear programming," International Journal of Electrical Power \& Energy Systems, vol. 42, pp. 299-305, 11// 2012.

[25] V. A. Papaspiliotopoulos, T. A. Kurashvili, and G. N. Korres, "Optimal coordination of directional overcurrent relays in distribution systems with distributed generation based on a hybrid PSO-LP algorithm," in MedPower 2014, 2014, pp. 1-6.

[26] X. Chunlin, Z. Xiufen, Y. Rongxiang, and W. Chuansheng, "Optimal coordination of protection relays using new hybrid evolutionary algorithm," in Evolutionary Computation, 2008. CEC 2008. (IEEE World Congress on Computational Intelligence). IEEE Congress on, 2008, pp. 823-828.

[27] A. Ahmarinejad, S. M. Hasanpour, M. Babaei, and M. Tabrizian, "Optimal Overcurrent Relays Coordination in Microgrid Using Cuckoo Algorithm," Energy Procedia, vol. 100, pp. 280-286, 11// 2016.

[28] H. R. E. H. Bouchekara, M. Zellagui, and Ma.Abido, "Optimal Coordination of Directional Overcurrent Relays Using a Modified Electromagnetic Field Optimization Algorithm," Applied Soft Computing.

[29] N. Mancer, B. Mahdad, K. Srairi, M. Hamed, and B. Hadji, "Optimal Coordination of Directional Overcurrent Relays Using PSO-TVAC," Energy Procedia, vol. 74, pp. 1239-1247, 8// 2015.

[30] P. P. Bedekar and S. R. Bhide, "Optimum Coordination of Directional Overcurrent Relays Using the Hybrid GA-NLP Approach," IEEE Transactions on Power Delivery, vol. 26, pp. 109-119, 2011.

[31] D. S. Alkaran, M. R. Vatani, M. J. Sanjari, G. B. Gharehpetian, and M. S Naderi, "Optimal Overcurrent Relay Coordination in Interconnected
Networks by Using Fuzzy-Based GA Method," IEEE Transactions on Smart Grid, vol. 9, pp. 3091-3101, 2018.

[32] Y. Damchi, H. R. Mashhadi, J. Sadeh, and M. Bashir, "Optimal coordination of directional overcurrent relays in a microgrid system using a hybrid particle swarm optimization," in Advanced Power System Automation and Protection (APAP), 2011 International Conference on, 2011, pp. 1135-1138.

[33] A. Sahoo and S. Chandra, "Multi-objective Grey Wolf Optimizer for improved cervix lesion classification," Applied Soft Computing, vol. 52, pp. 64-80, 3// 2017.

[34] M. H. Sulaiman, Z. Mustaffa, M. R. Mohamed, and O. Aliman, "Using the gray wolf optimizer for solving optimal reactive power dispatch problem," Applied Soft Computing, vol. 32, pp. 286-292, 7// 2015.

[35] H. Mee Song, M. H. Sulaiman, and M. R. Mohamed, "An Application of Grey Wolf Optimizer for Solving Combined Economic Emission Dispatch Problems," 2014, vol. 7, 2014.

[36] S. Mirjalili, S. M. Mirjalili, and A. Lewis, "Grey Wolf Optimizer," Advances in Engineering Software, vol. 69, pp. 46-61, 3// 2014.

[37] V. N. Rajput, K. S. Pandya, and K. Joshi, "Optimal coordination of Directional Overcurrent Relays using hybrid CSA-FFA method," in Electrical Engineering/Electronics, Computer, Telecommunications and Information Technology (ECTI-CON), 2015 12th International Conference on, 2015, pp. 1-6.

[38] "IEC standard 60255-4, Single input energizing measuring relays with dependent specified time.," in IEC standard 60255-4, ed: IEC publication 60255-4, 1st ed., 1976.

[39] N. Mittal, U. Singh, and B. S. Sohi, "Modified Grey Wolf Optimizer for Global Engineering Optimization," Applied Computational Intelligence and Soft Computing, vol. 2016, p. 16, 2016.

[40] A. J. Urdaneta, R. Nadira, and L. G. Perez Jimenez, "Optimal coordination of directional overcurrent relays in interconnected power systems," IEEE Transactions on Power Delivery, vol. 3, pp. 903-911, 1988.

[41] G. U. Darji, M. J. Patel, V. N. Rajput, and K. S. Pandya, "A tuned cuckoo search algorithm for optimal coordination of Directional Overcurrent Relays," in Power and Advanced Control Engineering (ICPACE), 2015 International Conference on, 2015, pp. 162-167. 